\title{
Biologists forced to reassess embryo test
}

\section{MONTREAL}

A technique for detecting genetic abnormalities in embryos is itself coming under the microscope. Preimplantation genetic diagnosis (PGD) is used in fertility treatment to check the condition of an embryo before it is implanted in a mother's womb. But studies now suggest that there is still much to learn about the procedure.

The findings suggest that genetically abnormal' embryos could still give rise to normal embryonic stem-cell lines. And they lend urgency to a newly launched effort to track the safety of the procedure, which is becoming increasingly popular but is regulated differently around the world.

In PGD, a cell is taken from the embryo at the eight-cell stage and tested for genetic abnormalities. If the cell is given the all-clear, the embryo is implanted in the mother's uterus. Babies born after the procedure seem to be fine, but few data have been collected on the children later in life.

It is also difficult to know how well the technique works in different cases. PGD is currently targeted at older women trying to have a baby. But whether it actually cuts the rate of miscarriage among these prospective mothers is only now being examined, researchers told a joint meeting of the American Society for Reproductive Medicine and the Canadian Fertility and Andrology Society in Montreal this week.

And results presented at the meeting show that PGD picks up surprisingly high rates of genetic abnormalities in younger women who donate eggs to infertile couples. That led some specialists, such as Jeffrey Nelson of the Huntington Reproductive Center in Pasadena, California, to suggest that the technique should be used more widely. "You can make an argument that PGD should be done in younger women", not just older women, he says.

But the most intriguing data presented at the conference suggest that embryos diagnosed as abnormal might be able to correct their genetic defects as they grow. A team including Santiago Munné of Reprogenetics, a company specializing in PGD based in West Orange, New Jersey, grew 55 embryos previously diagnosed as abnormal to the preimplantation stage, called the blastocyst. They

found that many of the embryos' cells were genetically normal. One embryo contained $76 \%$ normal cells after 12 days in culture. On average, $48 \%$ of the embryos' cells were genetically normal by the blastocyst stage.

These results hint that such embryos might be an 'ethical' source of embryonic stem cells for use in research. Normally, producing these cells means destroying a viable embryo, leading many groups to brand the practice as unacceptable (see page 1076). But the genetic abnormalities of embryos rejected by PGD mean that they are unlikely to develop into a healthy baby. ${ }^{\circ}$ These results suggest that we could get normal stem cells from abnormal embryos," says Munné. “These embryos will never implant, so this is ethically the best way of making them."

But the results also raise questions about whether cells that are not directly sampled by PGD are actually defective. If they aren't, the potential of these embryos remains undear. ${ }^{\alpha}$ These are the really important data we have to get from PGD," says Paulette Browne, a reproductive endocrinologist at the Shady Grove Fertility Center in Virginia. "It could be that some of the cells are abnormal and some are not, and we're starting to collect the rest of those data right now."

Together, the latest studies highlight the importance of a new effort to accumulate data on PGD, described at this week's meeting. Scientists and policy analysts announced the creation of a US database to track the safety of the procedure. A similar database exists in Europe, where PGD is tightly regulated, but US clinics, which carry out thousands of procedures, are not required to report their data.

The US database, set up by the Johns Hopkins University's Genetics and Public Policy Center in Washington DC, will collect information on who is using the technique in the country, the reasons for its use, and whether it results in healthy children.

Erika Check

\section{China launches plans for space exploration as taikonauts touch down}

The crew of China's second manned spacemission returned safely to Earth on 16 October, boosting the country's reputation as a spacefaring nation.

"This proves they are now a player to stay," says Joan JohnsonFreese, an expert on China's space programmeat the US Naval War College in Newport, Rhode Island. Economic development, education and national pride are important motivating factors, but the mission also serves as a global advertisement of China's technological capability, Johnson-Freese says.

The launch follows China's first manned mission in October 2003 , when Yang Liwei spent 21 hours in

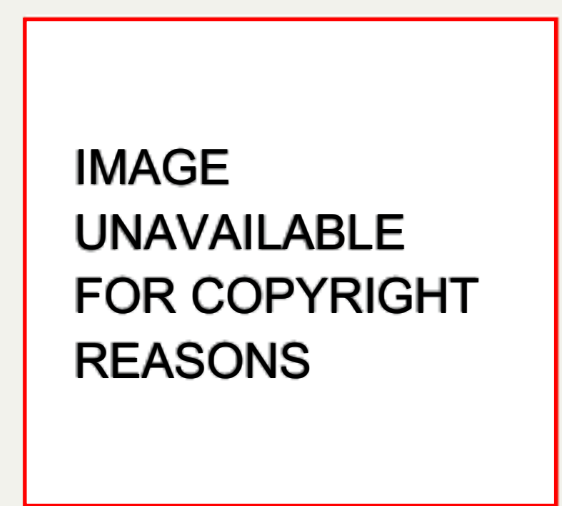

Fei Junlong (left) and Nie Haisheng arrive back from their five-day trip in space.

orbit. This mission was more ambitious, with two 'taikonauts' Fei Junlong and Nie Haisheng - spending five days in orbit in their Shenzhou craft, which is a close copy of the Russian Soyuz capsule. During themission, the taikonauts took off their space suits to conduct undisclosed scientific experiments in a habitation module attached to their small re-entry capsule.

Shortly after they landed in Inner Mongolia, the director of the

Manned Space Engineering Office, Tang Xianming, outlined plans for the nextmission. "Our estimate is that around 2007 our astronauts will walk in space," Tang told the state-run newspaper ChinaDaily.

Chinese delegates to an international conference on lunar exploration in Toronto, Canada, lastmonth also outlined near-term plans for robotic missions to the moon, including the Chang' $\mathrm{E}$ scientific orbiter set to launch in 2007. "China will beactive in humanity's exploration of unknown space," Hao Xifan of the Beijing-based China National Space Administration told the conference. He did not comment on Chinese plans to send astronauts to the Moon. Mark Peplow and Tony Reichhardt 